\title{
Low complexity FFT based spectrum sensing in Bluetooth system
}

\author{
Dong-Chan Oh and Yong-Hwan Lee \\ School of Electrical Engineering and INMC Seoul National University, Seoul, Korea \\ Kwan-Ak P.O. Box 34, Seoul, 151-600 Korea \\ Email: E-mail: mac81@ttl.snu.ac.kr, ylee@snu.ac.kr
}

\begin{abstract}
In this paper, we propose a complexity reduced spectrum sensing technique for Bluetooth to find channels free from the use of other communication devices in $2.4 \mathrm{GHz}$ ISM band. By exploiting the spectrum characteristics of interference sources, the proposed scheme detects the availability of channels by comparing the power spectrum density (PSD) with a threshold. To reduce the implementation complexity, the PSD is calculated by means of fast Fourier transform and linear interpolation. The threshold for the detection is determined to maximize the detection probability. To further improve the transmission performance, the proposed scheme dynamically changes the channels by measuring the transmission performance.
\end{abstract}

\section{INTRODUCTION}

As ubiquitous multimedia services are getting popular, the use of wireless local area network (WLAN) and personal area network (WPAN) are also widely considered. IEEE 802.11x based WLANs have been widely deployed to support wireless services in a moderately sized geographic area such as a single building or campus [1]. The use of Bluetooth is also widely spread out as an important element of WPANs. These WPAN and WLAN systems are often operating in unlicensed industrial, scientific, and medical (ISM) frequency band for low deployment cost. As a consequence, interference from coexisting devices is unavoidable. Recent works have shown that the performance of Bluetooth piconet can severely be degraded due to interference from collocated piconets or other heterogeneous wireless devices [2], [3]. Especially, recent measurement study reported that most of packet losses in a WPAN system are due to the interference of 802.11 WLAN [4].

For coexistence with other heterogeneous systems in 2.4 $\mathrm{GHz}$ ISM band, Bluetooth employs a pseudo-random frequency hopping (RFH) technique [5]. The RFH tries to mitigate interference by utilizing channels selected in a pseudorandom manner. However, it can only guarantee minimum packet error rate (PER) performance since it generates the hopping sequence without considering the characteristics of other system interference. This problem can be alleviated by employing an adaptive frequency hopping (AFH) developed by Task Group 2 (TG2) in IEEE 802.15 [6]. The AFH is processed in a two-step manner; channel classification and adaptive control action. Channel classification estimates the channel condition to detect the presence of any interference

\footnotetext{
${ }^{1}$ This work was supported by the Seoul R\&BD Program (10544)
}

source nearby by measuring the PER. Adaptive control action mitigates interference sources based on the channel classification result. The AFH can outperform the RFH by only utilizing channels in good condition. However, it is required to blindly transmit packets for the channel classification, causing possible packet loss and PER performance degradation.

Problems with the use of conventional RFH and $\mathrm{AFH}$ techniques can be alleviated by means of efficient spectrum sensing. The spectrum sensing is often achieved by three detection techniques: energy detection, matched filter coherent detection, and cyclo-stationary feature detection [7]. Since non-coherent energy detection is simple and is able to quickly locate the spectrum occupancy information, it is widely used for the spectrum sensing. Previous works considered the energy detection by cooperating among multiple radios [8], [9], but they can be applied to the detection of signals in a single channel. The signal detection in multiple channels can be achieved by estimating the power spectrum density (PSD) of a wideband signal. The PSD of a wideband signal can be estimated by means of fast Fourier transform (FFT). The existence of interference was detected by approximating the PSD of each channel as a Gaussian model [10]. However, it may take a long time to get a desired PSD from the FFT results. When applied to Bluetooth, it may need to perform FFT for all Bluetooth channels, resulting in huge computational complexity.

In this paper, we propose a complexity reduced FFT based spectrum sensing scheme to detect the existence of WLAN signal. The proposed scheme performs the FFT exactly once only for selected channels to cope with rapidly varying wireless channel environment. The PSD of other channels can be estimated by a simple linear interpolation technique. Since the proposed scheme performs the FFT only once, the PSD of each channel cannot be approximated as a Gaussian model. In the propose scheme, it is described by using a probability density function (pdf) of each channel condition (i.e., busy or idle). The threshold for the spectrum sensing is determined by a maximum a posteriori probability (MAP) criterion to maximize the detection probability. The rest of this paper is organized as follows. Section II describes the system model in consideration. The proposed scheme for the mitigation of interference is described in Section III. Section IV verifies the performance of the proposed scheme by computer simulation. Finally, conclusions are given in Section V. 


\section{SYSTEM MOdEL}

\section{A. Overview of IEEE 802.15.1 Bluetooth}

Bluetooth employs a frequency hopping/time division duplex (FH/TDD) scheme, where the channel is divided into consecutive slots. Each slot lasting $625 \mu \mathrm{sec}$ utilizes different hopping channel, yielding a nominal hop rate of 1600 hops/s. One packet can be transmitted per slot.

The channel makes use of several, equally spaced $1-\mathrm{MHz}$ hop channels. Bluetooth utilizes 79 channels in the $2.4 \mathrm{GHz}$ ISM band, each of which is equally used for the hopping in an average sense. It employs Gaussian-shaped frequency shift keying (GFSK) modulation, yielding a symbol rate of 1 Mbps. When 2-level GFSK is employed with non-coherent demodulator, it has a bit error rate given by [11]

$$
P_{B E}=\frac{1}{2} \exp \left(-\frac{\gamma}{2}\right)
$$

where $\gamma$ is the signal-to-noise ratio (SNR) per bit.

The corresponding packet error rate (PER) is represented as

$$
P_{P E}\left(N_{b i t s}, \gamma\right)=1-\left(1-P_{B E}\right)^{N_{b i t s}}
$$

where $N_{b i t s}$ is the number of bits in payload.

Bluetooth can provide voice services as well as data services. The voice is encoded into a 10-byte stream every 1.25 $\mathrm{ms}$. Since Bluetooth can send 30 bytes for each slot, it is required for real-time voice services to reserve a slot in both directions at every $3.75 \mathrm{~ms}$, which is called synchronous (SCO) link. It also has an asynchronous data link (ACL) for data transmission between the master and a slave. Since the SCO link has a priority over the ACL, the ACL can use only unused slots. The ACL has a single slot packet of 27 bytes, achieving a data rate of $172.8 \mathrm{Kbps}$ when packet is used. However, the presence of an SCO link significantly reduces the achievable data throughput of an ACL to 148.1 Kbps.

\section{B. Wideband Spectrum Sensing}

The received signal $r[n]$ in time domain can be represented as

$$
r[n]=s[n]+v[n]
$$

where $n$ denotes the sample index, $s[n]$ denotes the signal from other systems, and $v[n]$ denotes zero-mean additive white Gaussian noise (AWGN) with variance $\sigma_{v}^{2}$ (i.e., $v[n] \sim$ $\left.\mathcal{C N}\left(0, \sigma_{v}^{2}\right)\right)$. The corresponding signal at channel $k$ can be represented in the frequency domain by means of discrete Fourier transform (DFT) as

$$
\begin{aligned}
R[k] & =\frac{1}{\sqrt{N}} \sum_{n=0}^{N-1} r[n] e^{-j \frac{2 \pi n k}{N}} \\
& =S[k]+V[k], \quad k=0,1, \cdots, N-1
\end{aligned}
$$

where $S[k]$ and $V[k]$ are the DFT of $s[n]$ and $v[n]$, respectively. Without loss of generality, it can be assumed that $S[k]$ and $V[k]$ are independent of each other. In what follows, we consider the use of FFT instead of DFT for efficiency of computational complexity. To decide whether channel $k$ is occupied by other systems, consider the following simple hypothesis problem defined as

$$
R[k]=\left\{\begin{array}{cc}
V[k] & , \mathcal{H}_{0, k} \\
S[k]+V[k] & , \mathcal{H}_{1, k}
\end{array}\right.
$$

where $\mathcal{H}_{0, k}$ and $\mathcal{H}_{1, k}$ denote hypothesis corresponding to the absence (or idle state) and presence (or busy state) of other systems' signal in channel $k$, respectively. For each channel $k$, the existence of other systems' signal can be detected by comparing the corresponding PSD with a threshold value $\lambda$ as

$$
Y[k] \triangleq|R[k]|^{2} \underset{\mathcal{H}_{1, k}}{\stackrel{\mathcal{H}_{0, k}}{\lessgtr}} \lambda
$$

\section{Proposed Channel Sensing}

To improve the channel sensing performance, we exploit the spectrum characteristics of interference sources operating in $2.4 \mathrm{GHz}$ ISM band. The interference sources can be classified into three types, frequency-static interference, frequencydynamic self-interference, and channel noise. The frequencystatic interference occurs over a group of channels for a time interval much longer than the duration of a Bluetooth packet (e.g., IEEE 802.11x WLAN and IEEE 802.15.4 ZigBee). The frequency-dynamic self-interference is intermittent disturbance experienced from a collocated FH-based piconet that has used an identical channel simultaneously. Since most of packet losses in WPAN system is due to the interference of 802.11 WLAN [4], we mainly focus on the mitigation of WLAN interference in this paper. WLAN has wideband spectrum signal compared to Bluetooth. Therefore, if one of the Bluetooth channels is interfered by WLAN, its adjacent channels are also likely to be interfered. By taking into account this characteristic of other system interference, we design a low complexity FFT based spectrum sensing strategy to mitigate interference.

It is desirable to detect the existence of interference by performing the spectrum sensing before the signal transmission. It is known that receiver centric spectrum sensing works better than transmitter centric spectrum sensing in cellular environments [12]. However, since the communication range of Bluetooth is much shorter than that of cellular systems, both the Bluetooth master and slaves experience similar interference effect. Considering the power consumption and processing protocol for the spectrum sensing, it may be practical to make the Bluetooth master perform the spectrum sensing.

The proposed scheme performs the FFT one time for selected channels to reduce the computational complexity. Before the FFT, it is required to select $L$ Bluetooth channels equally separated by $s_{f}$ channels; called selected channels. For each selected channel, the master performs $N$-point FFT as

$$
R\left[s_{f} l\right]=\frac{1}{\sqrt{N}} \sum_{n=0}^{N-1} r[n] e^{-j \frac{2 \pi n s_{f} l}{N}}
$$

and calculates the PSD as

$$
Y\left[s_{f} l\right]=\left|R\left[s_{f} l\right]\right|^{2}
$$


where $l=0,1, \cdots, L-1$

The PSDs of the rest channels, called unselected channels, can be estimated by means of interpolation. To reduce the complexity, we consider the use of a simple linear interpolation algorithm as [13]

$$
Y\left[s_{f} l+i\right]=\alpha_{i} Y\left[s_{f} l\right]+\beta_{i} Y\left[s_{f}(l+1)\right]
$$

where $i=1,2, \cdots, s_{f}-1$, and $\alpha_{i}$ and $\beta_{i}$ are linear interpolation weights defined as

$$
\left\{\begin{array}{l}
\alpha_{i}=1-\frac{i}{s_{f}} \\
\beta_{i}=\frac{i}{s_{f}}
\end{array}\right.
$$

Finally, the master compares the PSD of channels with a threshold value $\lambda$ to detect the presence of interference. To compute the PSD of all channels by the $N$-point FFT, it requires $(N / 2) \log _{2} N$ complex multiplications and $N \log _{2} N$ complex addition. On the other hand, the proposed scheme requires $\left(N / 2 s_{f}\right) \log _{2} N+2(N-L)$ complex multiplication and $\left(N / s_{f}\right) \log _{2} N+(N-L)$ complex additions. For example, for $N=128$, the computational complexity for the multiplication and addition is reduced by $59 \%$ and $78 \%$, respectively.

The threshold value $\lambda$ can be determined from the conditional pdf for each hypothesis. It can easily be shown that the PSD $Y[k]$ has conditional pdf for each hypothesis as

$$
\left\{\begin{array}{l}
P_{Y[k]}\left(y_{k} \mid \mathcal{H}_{0, k}\right)=\frac{1}{\sigma_{v}^{2}} \exp \left(-\frac{y_{k}}{\sigma_{v}^{2}}\right) \\
P_{Y[k]}\left(y_{k} \mid \mathcal{H}_{1, k}\right)=\frac{1}{\sigma_{v}^{2}} \exp \left(-\frac{y_{k}+|S[k]|^{2}}{\sigma_{v}^{2}}\right) I_{0}\left(\frac{2|S[k]| \sqrt{y_{k}}}{\sigma_{v}^{2}}\right)
\end{array}\right.
$$

where $I_{0}(\cdot)$ is a modified Bessel function of the first of order zero.

The mean and variance of $Y[k]$ can be represented as

$$
E\{Y[k]\}= \begin{cases}\sigma_{v}^{2} ; & \mathcal{H}_{0, k} \\ \sigma_{v}^{2}+|S[k]|^{2} ; & \mathcal{H}_{1, k}\end{cases}
$$

and

$$
\operatorname{var}\{Y[k]\}= \begin{cases}\sigma_{v}^{4} ; & \mathcal{H}_{0, k} \\ \left(\sigma_{v}^{2}+2|S[k]|^{2}\right) \sigma_{v}^{2} ; & \mathcal{H}_{1, k}\end{cases}
$$

respectively.

Under hypothesis $\mathcal{H}_{0, k}$, the false detection probability is

$$
\begin{aligned}
p_{f}(\lambda) & =\operatorname{Pr}\left(Y[k]>\lambda \mid \mathcal{H}_{0, k}\right) \\
& =\exp \left(-\frac{\lambda}{\sigma_{v}^{2}}\right)
\end{aligned}
$$

Similarly, under hypothesis $\mathcal{H}_{1, k}$, the miss detection probability is

$$
\begin{aligned}
& p_{m}(\lambda)=\operatorname{Pr}\left(Y[k]<\lambda \mid \mathcal{H}_{1, k}\right) \\
& =\int_{0}^{\lambda} \frac{1}{\sigma_{v}^{2}} \exp \left(-\frac{y_{k}+|S[k]|^{2}}{\sigma_{v}^{2}}\right) I_{0}\left(\frac{2|S[k]| \sqrt{y_{k}}}{\sigma_{v}^{2}}\right) d y_{k}
\end{aligned}
$$

It can be shown from the (13) and (14) that the true detection probability for each hypothesis is

$$
\left\{\begin{array}{l}
p_{d}(\lambda)=1-p_{m}(\lambda) ; \mathcal{H}_{1, k} \\
p_{a}(\lambda)=1-p_{f}(\lambda) ; \mathcal{H}_{0, k}
\end{array}\right.
$$

Assuming that the packets are transmitted only when the master determines the channel is idle, the average opportunistic throughput at Bluetooth channel $k$ can be represented as

$$
\begin{aligned}
R(\lambda) & =\operatorname{Pr}\left(\mathcal{H}_{0, k}\right) N_{\text {bits }}\left[1-P_{f}(\lambda)\right]\left[1-P_{P E}\left(N_{b i t s}, \gamma_{N}\right)\right] \\
& +\operatorname{Pr}\left(\mathcal{H}_{1, k}\right) N_{b i t s} P_{m}(\lambda)\left[1-P_{P E}\left(N_{b i t s}, \gamma_{I N}\right)\right]
\end{aligned}
$$

where $N_{\text {bits }}$ is the number of bits per packet, $\operatorname{Pr}\left(\mathcal{H}_{1, k}\right)$ is the a priori probability that channel $k$ is occupied by other signal (i.e., interference), $\operatorname{Pr}\left(\mathcal{H}_{0, k}\right)=1-\operatorname{Pr}\left(\mathcal{H}_{1, k}\right)$, $P_{P E}\left(N_{b i t s}, \gamma_{N}\right)$ and $P_{P E}\left(N_{b i t s}, \gamma_{I N}\right)$ are the packet error rate due to the noise and the noise plus interference which can be derived from (2), respectively.

We determine the threshold value $\lambda$ to maximize the detection probability. With this goal in mind, we consider a decision rule based on the computation of posterior probabilities defined as $\operatorname{Pr}\left(\mathcal{H}_{m, k} \mid Y[k]\right)$, where $m=0,1$. The decision rule is based on selecting hypothesis (i.e., $\mathcal{H}_{0, k}$ or $\mathcal{H}_{1, k}$ ) corresponding to the maximum of the set of posterior probabilities $\operatorname{Pr}\left(\mathcal{H}_{m, k} \mid Y[k]\right)$. This decision criterion is called the maximum a posteriori probability (MAP) criterion.

Using the Bayer'rule, the posteriori probability can be represented as

$$
\operatorname{Pr}\left(\mathcal{H}_{m, k} \mid Y[k]\right)=\frac{\operatorname{Pr}\left(Y[k] \mid \mathcal{H}_{m, k}\right) \operatorname{Pr}\left(\mathcal{H}_{m, k}\right)}{\operatorname{Pr}(Y[k])}
$$

where $\operatorname{Pr}(Y[k])$ can be represented as

$$
\operatorname{Pr}(Y[k])=\sum_{m=0}^{1} \operatorname{Pr}\left(Y[k] \mid \mathcal{H}_{m, k}\right) \operatorname{Pr}\left(\mathcal{H}_{m, k}\right)
$$

Note that $\operatorname{Pr}(Y[k])$ is independent of the existence of interference in channel $k$. Consequently, the decision rule maximizing $\operatorname{Pr}\left(\mathcal{H}_{m, k} \mid Y[k]\right)$ is equivalent to maximizing $\operatorname{Pr}\left(Y[k] \mid \mathcal{H}_{m, k}\right) \operatorname{Pr}\left(\mathcal{H}_{m, k}\right)$. Therefore, the decision rule can be represented as

$$
\frac{\operatorname{Pr}\left(Y[k] \mid \mathcal{H}_{1, k}\right) \operatorname{Pr}\left(\mathcal{H}_{1, k}\right)}{\operatorname{Pr}\left(Y[k] \mid \mathcal{H}_{0, k}\right) \operatorname{Pr}\left(\mathcal{H}_{0, k}\right)} \underset{\mathcal{H}_{1, k}}{\stackrel{\mathcal{H}_{0, k}}{\lessgtr}} 1
$$

It can be shown from (10) that the decision rule (19) can be represented as

$$
\frac{\operatorname{Pr}\left(\mathcal{H}_{1, k}\right)}{\operatorname{Pr}\left(\mathcal{H}_{0, k}\right)} \exp \left(-\frac{|S[k]|^{2}}{\sigma_{v}^{2}}\right) I_{0}\left(\frac{2|S[k]| \sqrt{y_{k}}}{\sigma_{v}^{2}}\right) \underset{\mathcal{H}_{1, k}}{\stackrel{\mathcal{H}_{0, k}}{\lessgtr}} 1
$$

Therefore, the threshold value $\lambda$ can be determined as

$$
\lambda=\left[\frac{\sigma_{v}^{2}}{2|S[k]|} I_{0}^{-1}\left\{\frac{\operatorname{Pr}\left(\mathcal{H}_{0, k}\right)}{\operatorname{Pr}\left(\mathcal{H}_{1, k}\right)} \exp \left(\frac{|S[k]|^{2}}{\sigma_{v}^{2}}\right)\right\}\right]^{2}
$$

By comparing each estimated PSD with threshold value $\lambda$, the master determine the channel is idle or busy. After finding empty channels, the master generates an FH sequence for 32 empty channels.

The overall procedure of the proposed scheme is shown in Fig. 1. Packet losses can be occurred due to miss detection of interference signal. This problem can be alleviated by means of 


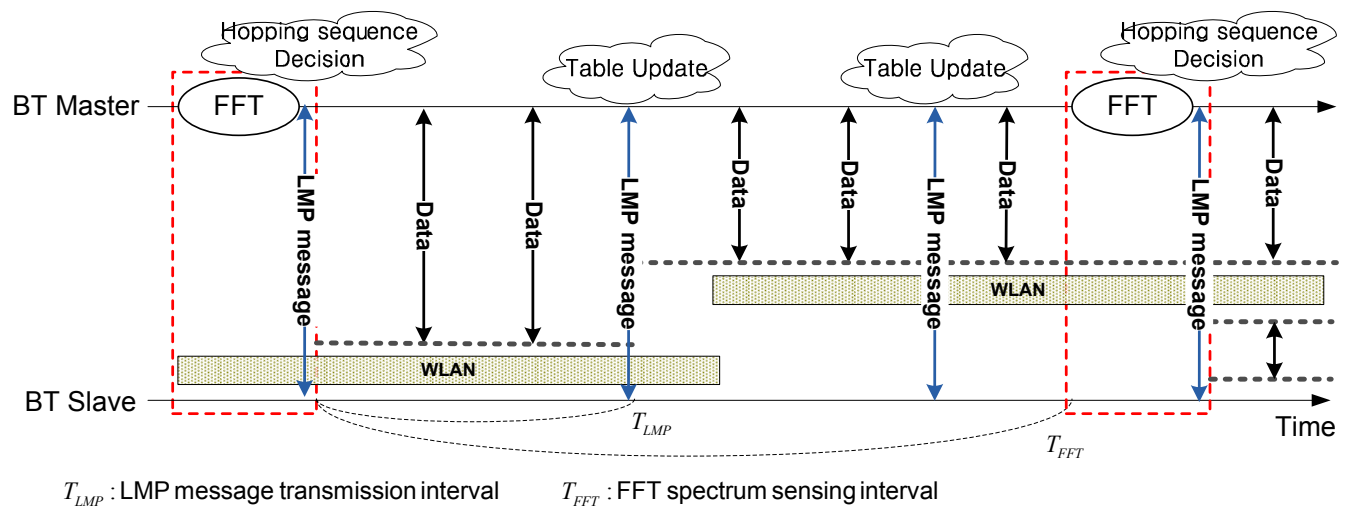

Fig. 1. Overall procedure of the proposed scheme.

TABLE I

COMMON SIMULATION PARAMETERS

\begin{tabular}{|c||c|}
\hline Simulation parameters & Setting \\
\hline Bluetooth master location & $(1,0)(m)$ \\
\hline Bluetooth slave location & $(0,0)(m)$ \\
\hline Bluetooth transmit power & $1 \mathrm{~mW}$ \\
\hline Bluetooth packet type & $\begin{array}{c}\text { DH1 for data ACL link } \\
\text { HV3 for voice SCO link }\end{array}$ \\
\hline LMP message feedback interval $\left(T_{L M P}\right)$ & 1000 packets \\
\hline FFT spectrum sensing interval $\left(T_{F F T}\right)$ & 10000 packets \\
\hline FFT size & 128 \\
\hline Number of selected frequency & 16 \\
\hline WLAN source location & $(0, d), d=15(m)$ \\
\hline WLAN transmit power & $25 \mathrm{~mW}$ \\
\hline Noise level & $-114 \mathrm{dBm}[15]$ \\
\hline Noise figure & $23 \mathrm{~dB}[15]$ \\
\hline
\end{tabular}

dynamic packet transmission and channel classification strategy based on a channel-by-channel classification method [14]. In this method, slaves in Bluetooth piconet calculate the PER for each channel. If the PER of a specific channel is higher than a desired PER, the slave requests the master the change of channel by using a link management protocol (LMP) message sent periodically with a period of $T_{L M P}$. Thus, the proposed scheme can transmit packets through only good channels while avoiding blind transmission as in the conventional RFH and AFH.

\section{Performance Evaluation}

The performance of the proposed scheme is verified by computer simulation. The common simulation parameters are summarized in Table 1.

Fig. 2 depicts the average opportunistic throughput of Bluetooth channel according to the change of threshold $\lambda$ in the presence of a single WLAN with offered load one. For fine representation, we scaled WLAN received power and noise level by Bluetooth received signal power. It can be seen that the analytic threshold value $\lambda$ from (21) (e.g., the derived threshold $\lambda=0.0378$ value for $d=5 \mathrm{~m}$ ) agrees

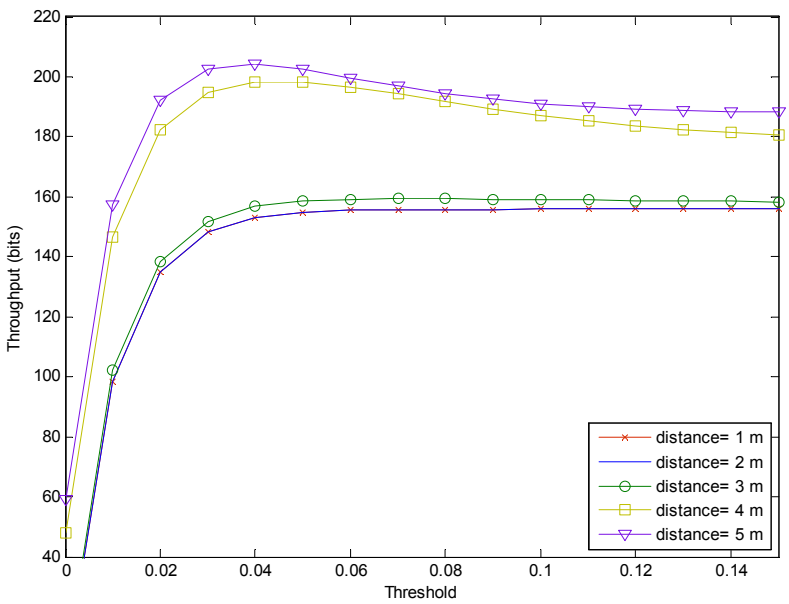

Fig. 2. Average opportunistic throughput per Bluetooth channel.

well with the simulation results. It can be also seen that when the distance from WLAN is short (e.g., less than 3 $\mathrm{m})$, the strength of interference is strong, yielding almost zero miss detection probability. As a consequence, the opportunistic throughput is almost the same. As the distance from WLAN increases, the opportunistic throughput also increases. This is due to the fact that as the distance increases, the effect of interference decreases, although the detection probability somewhat decreases.

Fig. 3 depicts the PER performance of the proposed Bluetooth system in the presence of one or two IEEE $802.11 \mathrm{~b}$ WLANs with various offered load factors (0.1-1) and distance $2 \mathrm{~m}$. It can be seen that as the offered load of WLAN increases, the proposed scheme can provide high performance gain over the conventional scheme that blindly transmit packets for channel classification. It can be also seen that the PER of the proposed scheme is slightly high when the activity of WLAN is medium. This can be explained as follows. When the activity of WLAN at channel $k$ is $q$, the Bluetooth master transmits packets through this channel with probability $1-q$. Thus, the probability that the Bluetooth packet collides with the WLAN 


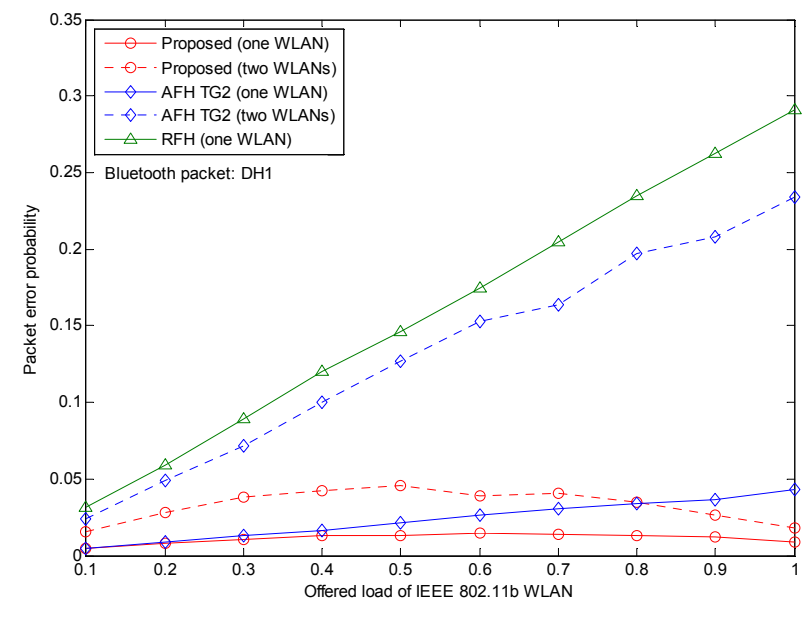

Fig. 3. PER performance of the proposed scheme.

signal is proportional to $(1-q) q$. As a consequence, the PER of the proposed scheme has a peak when the offered load of the WLAN is 0.5 . However, the proposed scheme significantly reduces the PER even in the presence of two WLANs.

Fig. 4 depicts the transmission rate of the proposed Bluetooth system when two WLANs with offered load 0.7 are working at distance $d$. It can be seen that the conventional AFH schemes is not working well when the distance is less than $4 \mathrm{~m}$, but the proposed scheme is little affected by the distance. It can also be seen that the data throughput is reduced when both voice and data traffic are in service. This is due to the fact that the SCO link has priority over the data transmission (i.e., the ACL can use only unused slots). As a consequence, the maximum achievable data rate of the ACL link is reduced from $172.8 \mathrm{Kbps}$ to $148.1 \mathrm{Kbps}$ due to the presence of an SCO link.

Although the proposed scheme outperforms conventional $\mathrm{RFH}$ and $\mathrm{AFH}$ schemes, it requires additional power to perform the FFT based spectrum sensing. A power consumption required to estimate PSD for all channels by performing 128-point FFT is about $16.2 \mathrm{~mW}$ [16]. However, since the proposed scheme reduces complexity about $40 \%$, the required power to perform spectrum sensing is about $10 \mathrm{~mW}$ which can be supported in Bluetooth specification in which supported power range is $0-100 \mathrm{~mW}$ [16].

\section{CONClusion}

In this paper, we have proposed a complexity reduced spectrum sensing technique for Bluetooth to detects channels being occupied by other communication devices in $2.4 \mathrm{GHz}$ ISM band. By exploiting the spectrum characteristic of the interference source and measuring the transmission performance, the proposed scheme can utilize idle channels for the packet transmission. The simulation results show that the proposed scheme is quite effective when the interference sources are located near the Bluetooth.

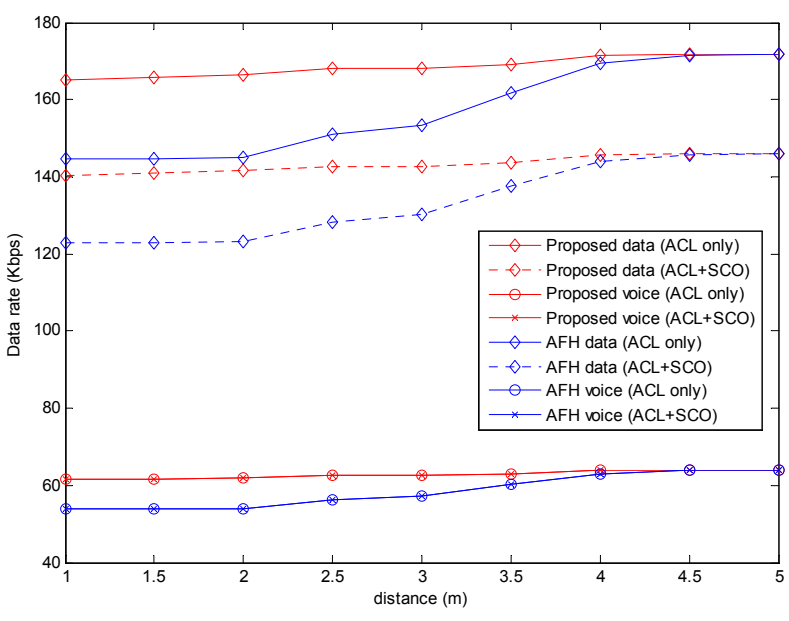

Fig. 4. Data rate of the proposed scheme.

\section{REFERENCES}

[1] IEEE 802.11b Specification, Wireless LAN Medium Access Control (MAC) and Physical Layer (PHY) Specifications: Higher-Speed Physical Layer Extension in the $2.4 \mathrm{GHz}$ Band, Sept. 16, 1999.

[2] N. Golmie, N. Chevrollier, and O. Rebala, "Bluetooth and WLAN coexistence: challenges and solutions," IEEE Wireless Commun., vol. 10, no. 6, pp. 22-29, Dec. 2003.

[3] P. Qixiang and V. C. M. Leung, "Channel Clustering and Probabilistic Channel Visiting Techniques for WLAN Interference Mitigation in Bluetooth Devices," IEEE Trans. Electromagn. Compat., vol. 49, no. 4, pp. 914-923, Nov. 2007.

[4] C.-W. Won, J.-H. Youn, H. Ali., H. Sharif, and J. Deogun, ”Adaptive radio channel allocation for supporting coexistence of 802.15.4 and 802.11b," in Proc IEEE VTC'05-Fall, vol. 4, pp. 2522-2526, Sept. 2005.

[5] Bluetooth Special Interest Group, Specification of the Bluetooth Systems, vol. 1, v.1.9B Core and 2, v1.0B, Dec 1999.

[6] IEEE 802.15 WPAN Task Group 2 (TG2), http://ieee802.org/15/pub/TG2.html, 2003.

[7] I. F. Akyildiz, W.-Y. Lee, M. C. Vuran, and S. Mohanty, "Next generation/dynamic spectrum access/cognitive radio wireless networks: a survey," Computer Networks, vol. 50, pp. 2127-2159, May 2006.

[8] D. Cabric, A. Tkachenko, and R. W. Brodersen, "Experimental study of spectrum sensing based on energy detection and network cooperation," in Proc. ACM Int. Workshop on TAPAS, Aug. 2006.

[9] S. Haykin, "Cognitive radio: Brain-empowered wireless communications," IEEE J. Select. Areas Commun., vol. 23, no. 2, pp. 201-220, Feb. 2005.

[10] Q. Zhi, C. Shuguang, A. H. Sayed, and H. V. Poor, "Wideband Spectrum Sensing in Cognitive Radio Networks," in Proc. IEEE ICC'08, pp. 901906, May 2008.

[11] J. G. Proakis and M. Salehi, emphCommunication Systems Engineering, Prentice Hall, New Jersey, 1994.

[12] J. Bater, H.-P. Tan, K. N. Brown, and L. Doyle, "Modeling Interference Temperature Constraints for Spectrum Access in Cognitive Radio Networks," in Proc IEEE ICC'07, pp. 6493-6498, June 2007.

[13] J.-H. Kim, J. H. Park, and D.-S. Hong, "Performance analysis of channel estimation in OFDM systems," IEEE Signal Process. Lett., vol. 12, no. 1, pp. 60-62, Jan. 2005.

[14] S.-H. Lee and Y.-H. Lee, "Novel frequency hopping and power control for interference mitigation in unlicensed communication systems," in Proc. UKC'08, Aug. 2008.

[15] J. C. Haartsen and S. Mattisson, "Bluetooth-a new low-power radio interface providing short-range connectivity," IEEE Proceedings, vol. 88, no. 10, pp. 1651-1661, Oct. 2000.

[16] Kazuto. N, Shingo. Y, and Yoshikazu. M, "A study of dynamic reconfigurable FFT Processor for OFDM based cognitive radio", in Proc. IEEE ISCIT, pp. 1507-1509, Oct. 2007. 